\title{
Study of Degree of Lumbar Wedging Measured Using CR- 35X Digitizer
}

\author{
Vinay $K V^{1 *}$ Vinay $H S,^{2}$ Vishal $K^{1}$
}

${ }^{1}$ Department of Anatomy, K.S. Hegde Medical Academy, Nitte University, Mangalore, Karnataka, India,

${ }^{2}$ Department of Orthopaedics, Sanjay Gandhi Institute of Trauma \& Orthopaedics, Bangalore, Karnataka, India.

\section{*Corresponding Author:}

Dr. Vinay Kumar $\mathrm{V}$

Assistant Professor, Department of Anatomy, K.S. Hegde Medical Academy, Nitte University, Deralakatte, Mangalore, Karnataka, India.

Email: drvinay66475@rediffmail.com

\section{Citation}

Vinay KV, Vinay HS, Vishal K. Study of degree of lumbar wedging measured using $\mathrm{cr}-35 \mathrm{x}$ digitizer. Nepal Journal of Medical Sciencs 2013;2(1):30-3.

\begin{abstract}
Background: Low back pain is a very common symptom. Population based normal variations of lumbar wedging (LW) in South Indian population is not well documented in literature. This study was done to compile a database of LW valid for healthy male and female subjects in the age range of 20-60 years in South Indian population.
\end{abstract}

Methods: The plain lateral radiographs of lumbar spine of 150 healthy subjects ( 88 males and 62 females) between 20 to 60 years were studied. The lumbar vertebral body height anteriorly (HBA) and posteriorly (HBP) were measured using CR-35X digitizer. The degree of LW was determined by the ratio of HBA/HBP.

Results: There was a gradual decrease in LW cranio-caudally from L1 to L5 in both sexes being maximum at L1 (0.96 in males and 0.97 in females) and minimum at L5 (1.13 in males and 1.13 in females). LW was higher in males than in females. LW is statistically significant only at L3 level and non significant at all other levels. Positive relationship of LW to age was not found.

Conclusion: The study provide the best available estimates of normal LW for the South Indian healthy subjects, which will be helpful to the clinicians, therapists and researchers as ready references to LW in South Indian population.

Keywords: lumbar wedging; lumbar vertebrae; vertebral body height.

\section{Background:}

T

he vertebral column of humans is unique among the animal species in both function and aging patterns. Humans are the only few species that engage in bipedal locomotive behaviour and the only species that uses bipedalism exclusively. Human lumbar vertebrae support the weight of the upper body. The vertebrae which are five bones are the largest vertebrae in dynamic part of the spinal column. Loads lifted and carried by the upper extremities cause significant loading stress to the vertebral bodies. In between the body of vertebrae are intervertebral discs made of fibrous cartilage that act as shock absorbers and allow the back to move. ${ }^{1}$

Back pain is an extremely common symptom, a price mankind has to pay for their upright posture. Low back 


\section{Original Article | Vinay KV et al. Study of degree of lumbar wedging}

ache, sciatica or nerve root compression has reached an epidemic proportion. It is a common cause of occupational and domestic disability in industrialized societies. The pattern of behaviour of the normal back and the nature of the events and incidents which lead to derangements have intrigued the practitioner since primitive times.

Back pain is a very common symptom in the population. The anterior wedging of the vertebrae in the lower thoracic and upper lumbar spine is closely related to complaints of backpain. The presence of one or several anteriorly wedged vertebrae in radiographs of the spine, is usually considered abnormal. ${ }^{2}$ A mild degree of anterior vertebral body wedging may be a normal developmental variant with no clinical significance. $^{3}$ Definitive criteria for the measurement of lumbar wedging (LW) appear to be lacking. Even though the problem of low backache is equally prevalent all over the universe, we see little studies done on LW in Indian context. It is well established that the morphometrical data varies within different sex, race, ethnic and regional groups. Population based normal variations of LW in South Indian population is not well documented in literature. Since the normal variations in LW are not known, the present study was undertaken. The main objective was to compile a database of LW valid for healthy male and female subjects in the age range of 20-60 years in South Indian population.

\section{Methods:}

Plain radiographs (lateral view) of the lumbar spine of 150 healthy subjects of known sex (88 males and 62 females) between age groups of 20 to 60 were examined in the present study. In order to avoid uncertainty of measurement caused by secondary ossification centres of vertebrae before 20 years and osteoporosis after 60 years, subjects between 20 to 60 years were selected. No individuals were again exposed to X-rays for this study, rather the radiographs were collected from the patient's records whose radiographs were taken as part of investigations for abdominal conditions or intravenous pyelography from the department of Radiology, K. S. Hegde Medical Academy and Kasturba Medical College, Mangalore, Karnataka, India. Only those subjects having no symptoms of the spine were selected. The radiographs were screened for readability and an attempt was made to eliminate subjects with significant vertebral anomalies and other problems likely to influence the growth and development. All the radiographs were taken in the lateral recumbent position with the hips and knees flexed to $45^{\circ}$. The X-ray beam was centered on L3 and an anodefilm distance of $100 \mathrm{~cm}$ was maintained. The magnification resulting from the use of this technique was negligible.
These radiographs were scanned by CR 35-X digitizer and then the following parameters were measured. The height of the vertebral body anteriorly (HBA) and posteriorly (HBA) were measured at a distance of $2 \mathrm{~mm}$ from the middle part of the borders of vertebral bodies to avoid edges of vertebral bodies (Figure -1).

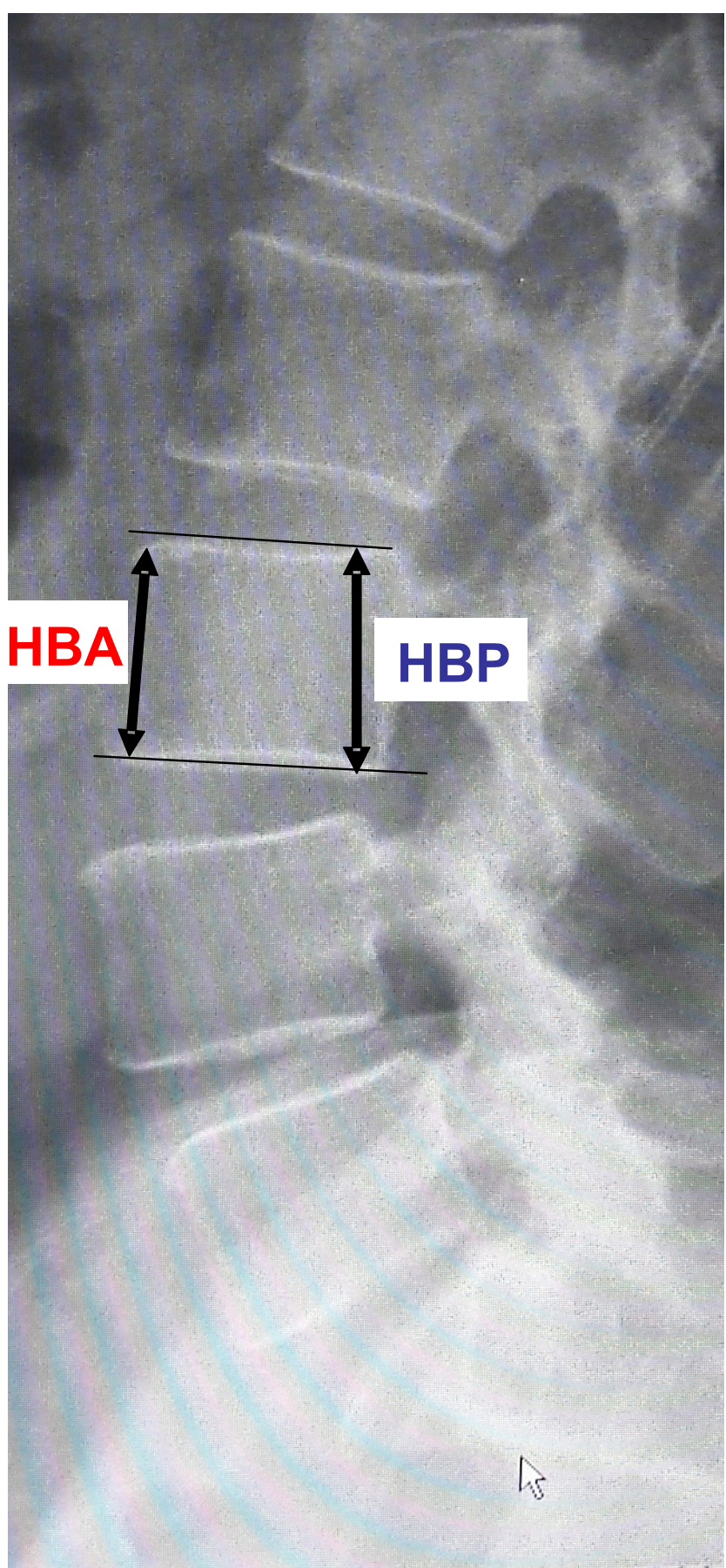

Figure 1. Lateral radiograph of the lumbar vertebrae illustrating the landmarks used for measuring the height of vertebral body anteriorly (HBA) and posteriorly (HBP).

The LW is determined by the ratio of HBA $/ \mathrm{HBP}^{3}$ This means that a high degree of LW corresponds to a small value 
of the ratio HBA/HBP. The mean and standard deviations of LW were calculated. Student t- test was used to compare LW between male and female subjects. The confidence limit was calculated and compared with the other studies.

\section{Results:}

The LW was determined by the ratio of HBA / HBP which means that a high degree of LW corresponds to a small value of the ratio HBA/HBP. ${ }^{4}$ The mean LW (Table-1) gradually decreases cranio-caudally from L1 to L5 in both male and female groups.

Table-1: Comparision of Lumbar wedging ( $L W)$ in males and females.

\begin{tabular}{ccccc}
\hline Lumbarlevel & Sex & MeanLW & $\begin{array}{c}\text { Standard } \\
\text { deviation }\end{array}$ & P value \\
\hline L1 & Male & 0.96 & 0.11 & 0.58 \\
& Female & 0.97 & 0.08 & \\
L2 & Male & 0.98 & 0.09 & 0.08 \\
& Female & 1.01 & 0.08 & \\
L3 & Male & 0.99 & 0.06 & 0.03 \\
& Female & 1.02 & 0.07 & \\
L4 & Male & 1.03 & 0.07 & 0.35 \\
& Female & 1.04 & 0.09 & \\
L5 & Male & 1.13 & 0.12 & 0.89 \\
& Female & 1.13 & 0.14 & \\
\hline
\end{tabular}

The maximum LW was at L1 level (0.96 in males and 0.97 in females) and minimum at L5 level (1.13 in males and 1.13 in females). The standard deviation was maximum at L5 level in both sexes. The degree of LW was greater in male group than female group at almost all vertebral level but it was statistically significant only at L3 level. Positive relationship to age was not found.

\section{Discussion:}

Wedging of the vertebral body seen on radiographs is one of the characteristic features in osteoporotic or traumatic compression fractures. The diagnosis of vertebral compression fractures is based on a history of trauma, clinical symptoms such as back pain, and radiological findings. Among them, one of the most reliable diagnostic parameters may be wedging of the vertebral bodies in the sagittal plane detected on radiographs, reconstruction $\mathrm{CT}$, and magnetic resonance imaging (MRI). Because the anterior spinal column fails in compression while the middle spinal column remains intact, the vertebral body becomes wedge-shaped. However, there have been several reports regarding physiological wedging of the thoracolumbar vertebral bodies in subjects without any history of trauma. ${ }^{4-9}$

Hurxthal $\mathrm{ML}^{10}$ studied lumbar vertebral bodies in 20 lateral radiographs which revealed that the HBP was more at L3, L4 and L5. At L1 and L2 levels HBA was more than HBP. In males, HBP decreases relatively to HBA so that the lumbar bodies become wedge shaped with age. The lumbar body becomes lower and broader with age. ${ }^{11}$ Although HBA and HBP varies with age, The ratio of HBA and HBP doesnot show any statistical significance. ${ }^{12}$

Lauridsen $\mathrm{KN}$ et al., ${ }^{4}$ studied vertebrae from T8 to L3 and reported that LW was greater at almost all levels in the male group than in the female group and the LW decreased cranio- caudally from T8 to L3. These observations were similar to our study. The mean LW observed by Lauridsen et al. was higher than the present study. The vertebrae L4 and $\mathrm{L} 5$ couldnot be compared as the study by Lauridsen $\mathrm{KN}$ et al., ${ }^{4}$ was done from T8 to L3 vertebrae. The confidence limits for $95 \%$ by Lauridsen $\mathrm{KN}$ et al., ${ }^{4}$ was $0.82,0.86$ and 0.90 in males and $0.86,0.90$ and 0.92 for L1, L2 and L3 respectively. In the present study the confidence limits for 95\% was 1.05, 1.08 and 1.09 in males and 1.09, 1.16 and 1.11 for L1, L2 and L3 respectively (Table 2). Vertebral wedging was more remarkable in men than in women ${ }^{13}$ which was similar to our study.

Table-2. Degree of Lumbar wedging (LW) with confidence limit $95 \%$.

\begin{tabular}{ccc}
\hline Vertebral level & Males & Females \\
\hline L1 & 1.05 & 1.09 \\
L2 & 1.08 & 1.16 \\
L3 & 1.09 & 1.11 \\
L4 & 1.16 & 1.24 \\
L5 & 1.29 & 1.31 \\
\hline
\end{tabular}

\section{Conclusion:}

This study evaluated the influence sex on LW. The degree of LW was greater in male group than female group at almost all vertebral level but it was statistically significant only at L3 level. No positive relationship to age was found. The findings of the present study will be helpful to the clinicians, therapists and researchers as ready references of LW in South Indian population. Any deviation from this value should be correlated with clinical findings.

\section{Conflict of interest: none}

\section{References:}

1. Moore KL. Clinically Oriented Anatomy. 3th Ed. Baltimore, Williams \& Wilkins, 1992:332. 
2. Lings S. Scheuermann's disease with low localization. Scand J Rehabil Med 1982:14;77-9.

3. Fletcher GH. Anterior vertebral wedging. Frequency and significance. Am J Roentgenol 1947;57: 232-8.

4. Lauridsen KN, De Carvalho A, Andersen AH. Degree of vertebral wedging of dorso lumbar spine. Acta Radiologica Diagnosis 1984;25:29-32.

5. Abdel-Hamid OA, Bassiouni H, Koutri R, et al. Aging of the thoracic spine: distinction between wedging in osteoarthritis and fracture in osteoporosis - a cross-sectional and longitudinal study. Bone 1994;15:437-42.

6. Davies KM, Recker RR, Heaney RP. Normal vertebral dimensions and normal variation in serial measurements of vertebrae. J Bone Miner Res 1989;4:341-9.

7. Diacinti D, Acca M, D'Erasmo E, et al. Aging changes in vertebral morphometry. Calcif Tissue Int 1995;57:426-9.
8. Evans S, Nicholson P, Haddaway M, et al. Vertebral morphometry in women aged 50-81 years. Bone Miner 1993;21:29-40.

9. Gallagher JC, Hedlund LR, Stoner S, et al. Vertebral morphometry: normative data. Bone Miner 1988;4:189-96.

10. Hurxthal ML. Measurement of anterior vertebral compressions and biconcave vertebrae. Am J Roentgenol 1968;103:635-44.

11. Ericksen MF. Some aspects of aging in the lumbar spine. Am J phys Anthropol 1976;45:575-80.

12. Gaca AM, Barnhart HX, Bisset GS. Evaluation of Wedging of Lower Thoracic and Upper Lumbar Vertebral Bodies in the Pediatric Population. Am J Roentgenol 2010;194:516-20.

13. Morio M. Wedging of vertebral bodies at the thoracolumbar junction in asymptomatic healthy subjects on magnetic resonance imaging. Surg Radiol Anat 2011;33:223-8. 